them. From a practical standpoint attention has been chiefly focussed on determining whether benign hyperplasia, as the commoner condition, plays any part in influencing the onset of malignancy. Some guidance on this point may be provided by the usual sites of the two diseases. Whereas benign hyperplasia arises in the inner periurethral group of prostatic glands, the consensus of histological opinion favours the outer zone of peripheral glands as the primary site of origin for cancer. Though it is not uncommon clinically for cancer to coexist with hyperplasia, the early malignant lesion is characteristically discrete, and the occasional chance detection of unsuspected cancer in an enucleated or resected "adenomatous prostate" may generally be ascribed to infiltration. Consequently simple enucleation prostatectomy-the removal of the benign adenomatous element-does not confer immunity from the possible later development of cancer in the residual peripheral remnant of the gland.

But can the presence of benign hyperplasia, by either its proximity or other local effect, predispose to malignant change in the remainder of the organ? Alternatively, could some unknown factor, possibly hormonal or immunological, account for both benign and malignant prostatic disease?

Fortunately neither possibility receives any support from the findings of a recent retrospective review undertaken under the auspices of the Lahey Clinic Foundation. ${ }^{1}$ Over 800 patients with benign prostatic hyperplasia were matched and compared with a similar series of controls over a period of more than 10 years. The average age of entry into the study was 63 years for both patients and controls, with $66 \%$ of both groups in the 60-69 year age group; $23 \%$ were below the age of 60 and $11 \%$ were 70 or over. Transurethral resection was performed in $89.5 \%$ of the patients with prostatic hyperplasia, suprapubic subtotal prostatectomy in $9.9 \%$, and perineal prostatectomy in $0.6 \%$. A condition for inclusion in the study was a pathological report on the resected tissue that was negative for cancer. In the event 24 patients (benign hyperplasia) and 26 controls subsequently developed cancer -a proportion corresponding closely to the natural expectancy of the disease according to age, and giving no indication of cause or effect.

If any criticism were to be made of the study it might be levelled at the justification of excluding cancer purely on the basis of histological examination of resected prostatic material. However, from the duration of the review and the stringent guidelines imposed, there seems little reason to doubt the validity of the findings, which confidently exclude benign enlargement of the prostate as either a premalignant condition or a cause of cancer.

${ }^{1}$ Greenwald, P., et al., fournal of the National Cancer Institute, 1974, 53, 335.

\section{Lumbar Puncture}

Quincke ${ }^{1}$ described the technique of lumbar puncture in 1891, brilliantly conceived for treating hydrocephalus and diagnosing tuberculous meningitis. Lumbar puncture is relatively safe because the spinal cord ends (in adults) at the level of the first lumbar vertebra. Nevertheless, it is often an ordeal for both physician and patient; the information gained may be slight or misleading, as when a bloody tap is wrongly interpreted as indicating subarachnoid haemorrhage; and the procedure is not without risks. These include respiratory arrest and sudden death in patients with raised intracranial pressure and catastrophic reactions to intrathecal antibiotics and other foreign substances. ${ }^{2}$ Iatrogenic meningitis and spinal abscess have been caused by lumbar puncture done in the presence of lumbosacral sepsis, while epidermoid spinal tumours may be associated with repeated punctures with an open needle in children. ${ }^{45}$ The catastrophe of spinal subdural haematoma with permanent paralysis has resulted from lumbar puncture in patients with coagulation defects, in whom this procedure should be done only for a compelling reason-and if necessary a prophylactic platelet transfusion should be given. ${ }^{6}$

The diagnosis of meningitis and the planning of its therapy require lumber puncture; Samson et al. ${ }^{7}$ found that over a third of children with purulent meningitis who had had a major motor seizure had no clinical evidence of meningitis, a fact which emphasizes the risks of not examining the cerebrospinal fluid. Management of many other neurological diseases also requires a lumbar puncture. Examples include the decision to give anticoagulants to a patient with a stroke in evolution and the pressure measurements which are of value in the investigation of possible spinal tumours - but the lumbar puncture may result in a partial paralysis becoming complete. ${ }^{8}$ Fringe benefits include determinations of brain enzymes and metabolites, though the concentration of substances which originate in cerebral as opposed to spinal tissues lprogressively diminishes from ventricular to cisternal to lumbar fluid. ${ }^{9}$

The risks of lumbar puncture in the presence of raised intracranial pressure are difficult to assess but may be considerable. Distortion of the brain-stem or supratentorial herniation may rapidly follow the procedure in patients with brain oedema due to such diverse causes as head injury and lead poisoning as well as cerebral tumour. Evidence of raised pressure is given by headache, mental changes, and progression of focal neurological signs; but papilloedema, pineal displacement, and clinoid erosion may all be absent. ${ }^{2}$ If clinical suspicion of a lesion in the posterior fossa is high,lumbar puncture will not alter management, and noninvasive techniques such as computer-assisted tomography ${ }^{10}$ will give more pertinent information. $)^{11}$

The most common complication of lumbar puncture is still headache due to slow leakage of cerebrospinal fluid around the dural tear. This defect can be minimized if the needle bevel is inserted in the plane of dural fibres, most of which run longitudinally. Position after puncture may be all-important; Brocker $^{12}$ studied 894 patients who lay prone and 200 supine afterlumbar puncture with a fairly large gauge (18) needle. The incidence of headache was $0.5 \%$ and $36.5 \%$ respectively, a difference which may have been due to the prone position causing misalignment of the holes in dura and arachnoid and thus reducing the leakage of cerebrospinal fluid. The decision to tap or not to tap is often difficult, but with care lumbar puncture should cause little distress and no hazard to the patient.

\footnotetext{
1 Quincke, H., 1891, in Neurosurgical Classics, ed. R. H. Wilkins. New York, Johnson Reprint Corporation, 1965.

Duffy, G. P., British Medical fournal, 1969, 1, 407.

British Medical fournal, 1970, 3, 412.

Rifaat, M., et al., Fournal of Neurosurgery, 1973, 38, 366

5 Shaywitz, B. A., Fournal of Pediatrics, 1972, 80, 638.

Edelson, R. N., Chernik, N. L., and Posner, J. B., Archives of Neurology, 1974, 31, 134

Samson, J.H., Apthorp, J., and Finley, A., fournal of the American Medical Association, 1969, $210,1918$.

${ }^{8}$ Bayliss, R. I. S., Practical Procedures in Clinical Medicine, 3rd edn. London, Churchill, 1960.

9oir, A. T. B., et al., Brain, 1970, 93, 357.

10 Moir, A. T. B., et al., Brain, 1970, 93,
10 British Medical fournal, 1974, 2, 623.

11 Petito, F., and Plum, F., New England fournal of Medicine, 1974, 290, 225

12 Betito, F., and Plum, F., New England Fournal of Medicine, 1974, 290, 225.
} 